\title{
Knowledge about Polycystic Ovarian Syndrome (PCOS) and Effect of Lifestyle Habits among Medical Students of North India
}

\author{
Monika Gupta ${ }^{1}$, Sartaj Ahmad², Chhavi Kiran Gupta ${ }^{3}$, Varsha Chaudhary ${ }^{4}$, Pawan Parashar ${ }^{4}$, \\ Ravi Sashtri ${ }^{5}$ \\ ${ }^{1}$ Professor, ${ }^{2}$ Associate Professor (Medical Sociology), ${ }^{3}$ Associate Professor, Department of Community \\ Medicine, Subharti Medical College, Swami Vivekananda Subharti University, Meerut UP India, ${ }^{4}$ Professor, \\ ${ }^{5}$ JR III, Department of Community Medicine, Subharti Medical College, Meerut UP India
}

\begin{abstract}
Introduction: Polycystic ovary syndrome (PCOS) is a heterogeneous, chronic endocrine disorder commonly diagnosed in women of reproductive age and the most common cause of hyperandrogenism and hirsutism.
\end{abstract}

Objective: To assess the knowledge about PCOS among medical students and to assess the awareness about effects of lifestyle habits on PCOS.

Material \& Method: This cross sectional study was done among100 undergraduate medical students of a private medical college of Western UP using a pre designed semi-structured questionnaire.

Results: Majority (84\%) of students were already aware about PCOS. Significant association was found between the awareness about PCOS with gender of study population. Majority ( $80 \%)$ of students were already aware about the lifestyle changes. There was significant association between knowledge about lifestyle modifications in PCOS with the knowledge about etiology of PCOS.

Conclusion: it is a growing endocrinological problem in the females of the reproductive age group. Early screening is necessary to prevent lifelong complications.

Key words: awareness, polycystic ovarian syndrome, medical students.

\section{Introduction}

Polycystic ovarian syndrome (PCOS) is the most prevalent endocrine disorder in women of reproductive age, and is the most common cause of

\section{Corresponding author:}

\section{Dr. Varsha Chaudhary,}

Professor, Department of Community Medicine,

Subharti Medical College, Swami Vivekananda

Subharti University, Meerut UP India.

Email :mahichaudhary25@gmail.com

Contact: 9897694695 hyperandrogenism and hirsutism. The prevalence of PCOS varies from $2.2 \%$ to $26 \%$ in different countries and has been reported as $7.1 \%$ based on National Institutes of Health $(\mathrm{NIH})$, criteria, and as $11.7 \%$ based on Androgen Excess Society (AES) criteria. [1] The exact cause of PCOS is unknown but experts believe that it is related to the production of excess amount of androgen (a group of male sex hormones). Although all women produce some androgens, too much of this hormone can cause PCOS. ${ }^{[2]}$ The mechanism of development of PCOS is unknown, but it can be attributed to various genetic factors 
which work in close approximation with various environmental factors. Some experts suggest that it may be an X-linked inheritance.

Menstrual irregularity is the most common manifestation of PCOS and is present in almost $80 \%$ of patients. The usual menstrual irregularities are oligomenorrhea or secondary amenorrhea and sometimes primary amenorrhea or menorrhagia. However, $20 \%$ of women may have apparently regular cycles despite an ovulation. hirsutism is present in 50$60 \%$, acne in $15-20 \%$, and androgenic alopecia in 5\% of patients with PCOS. Approximately 30-75\% of patients with PCOS are obese, and majority of them have an android distribution of fat, with features of insulin resistance Women with PCOS are more likely to suffer from depression, anxiety, poor self-esteem, alter the coping abilities, strain relationships, decrease quality of life, disordered eating and psychosexual dysfunction $^{[3]}$ Other problems linked to PCOS include sudden weight gain, sleep apnoea, diabetes, heart disease, hypertension, metabolic syndrome including hyperlipidemia and mood related disorders. ${ }^{[4]}$ Findings suggest that women with PCOS should be screened for mental health disorders to ensure early diagnosis and treatment and ultimately improve their quality of life. ${ }^{[5]}$ Lifestyle modification is the preferred first line treatment for PCOS. Nonpharmacological measures such as diet and exercise are recommended as first line of treatment in oligomenorrhoea, hirsutism, infertility, and obesity in PCOS by the majority of endocrinologists and gynecologists. ${ }^{[6]}$.

\section{Objectives}

_ To assess the knowledge about PCOS among medical students .

_ To assess the awareness about effects of lifestyle habits on PCOS.

_ To evaluate their perception and increase in awareness towards PCOS after intervention

\section{Material and Methods}

After the ethical consideration by the ethical committee of the department, the study was undertaken and data collection was conducted.

Study area - Subharti Medical College

Study design- cross sectional study

Sampling technique - Purposive sampling.

Study population- Undergraduate students of MBBS $1^{\text {st }} \& 2^{\text {nd }}$ professional year of Subharti Medical College

Sample size -100 students.

Research tool- Pre designed semi-structured questionnaire.

Inclusion \& Exclusion criteria- Students of MBBS $1^{\text {st }} \& 2^{\text {nd }}$ professional year who were present and willing to take part in the study were included and rest were excluded.

\section{Results and Findings}

Table 1 -Distribution Of Study Population according To The Awareness About PCOS

\begin{tabular}{|c|c|c|}
\hline $\begin{array}{c}\text { AWARENESS ABOUT } \\
\text { PCOS }\end{array}$ & $\begin{array}{c}\text { PRE INTERVENTION } \\
(\%)\end{array}$ & POST INTERVENTION \\
\hline Yes & 84 & 97 \\
\hline NO & 16 & 3 \\
\hline
\end{tabular}


Table 2 - Association Between Awareness About PCOS With Gender Of Study Population

\begin{tabular}{|c|c|c|}
\hline Gender & Present & Absent \\
\hline Male & $48(34.11 \%)$ & $03(05.89 \%$ \\
\hline Female & $36(73.40 \%)$ & $13(26.54 \%)$ \\
\hline
\end{tabular}

c $2=8.823, \mathrm{P}=0.003$

Table 3 - ASSOCIATION BETWEEN AWARENESS ABOUT PCOS AND FATHER'S EDUCATION

\begin{tabular}{|c|c|c|}
\hline FATHER'S EDUCATION & Present N=84 & Absent=16 \\
\hline $\begin{array}{c}\text { Primary to intermediate or post high } \\
\text { School diploma }\end{array}$ & $4(4.76 \%)$ & $1(6.25 \%)$ \\
\hline $\begin{array}{c}\text { Graduate or post graduate } \\
\text { Profession or honours }\end{array}$ & $55(65.47 \%)$ & $03(18.75 \%)$ \\
\hline
\end{tabular}

c $2=14.095, \mathrm{P}=0.0009$

Table 4 - Distribution Of The Study Population According To The Knowledge About Changes In PCOS

\begin{tabular}{|c|c|c|}
\hline $\begin{array}{c}\text { Knowledge } \\
\text { About changes } \\
\text { In PCOS }\end{array}$ & Pre Intervention & Post Intervention \\
\hline $\begin{array}{c}\text { Changes in menstrual } \\
\text { cycle }\end{array}$ & $48 \%$ & $78 \%$ \\
\hline Changes in body hair & $53 \%$ & $67 \%$ \\
\hline Changes in scalp hair & $51 \%$ & $64 \%$ \\
\hline
\end{tabular}

In this study majority (84\%) of students were already aware about PCOS and post presentation, there was a $13 \%$ increase in the awareness. Majority of males $(94.11 \%)$ and females $(73.46 \%)$ were aware about PCOS. Significant association was found between the awareness about PCOS with gender of study population.
The association between the awareness about PCOS and father's education. $4.76 \%$ of their fathers had primary to post high school level of education, $65.47 \%$ had graduate or post-graduate level of education, and $29.76 \%$ had professional or honors level of education. There is significant association between the awareness about PCOS and father's 
education.

The distribution of the study population according to the knowledge about the changes in PCOS. There was an initial knowledge of $48 \%$ about the changes in menstrual cycle, and post intervention there was an increase of $30 \%$. Knowledge about changes in body hair was 53\% and post intervention increase was of $14 \%$. Knowledge about changes in scalp hair was initially $51 \%$ with a post intervention increase of $13 \%$ about the same.

Table 5 - Association Between Awareness About Changes In PCOS with knowledge about etiology of PCOS

\begin{tabular}{|c|c|c|}
\hline $\begin{array}{c}\text { KNOWLEDGE } \\
\text { ABOUT } \\
\text { ETIOLOGY }\end{array}$ & AWARE (N=75) & NOT AWARE (N=25) \\
\hline PRESENT & $47(6266 \%)$ & $8(32 . .00 \%)$ \\
\hline ABSENT & $28(3733 \%)$ & $17(68.00 \%)$ \\
\hline
\end{tabular}

c $2=7.125, \mathrm{P}=0.0076$

The association between the awareness about changes in PCOS with the knowledge about the etiology of PCOS. Among the aware, in 62.66\% knowledge about etiology was present and in $37.33 \%$ it was absent. There is significant association between awareness about changes in PCOS with knowledge about etiology of PCOS. Majority (80\%) of students were already aware about the lifestyle changes and post presentation these was an increase of $8 \%$ in the knowledge about the positive effect of physical exercise in PCOS.

The distribution of the study population according to the knowledge about the lifestyle changes in regards with type of exercises for PCOS. Majority of the students were already aware about Yoga (77\%) and Walk/running in open (60\%) as a form of lifestyle change, and post presentation there was an increase of $7 \%$ in Yoga and 22\% in walk/running in open as an effective form of lifestyle change. There was an initial knowledge about gym, playing a sport and dieting as a form of lifestyle change, post presentation there was an increase in all three types of lifestyle changes.

The distribution of the study population according to the knowledge about the positive effect of diet on PCOS. Majority of the population had knowledge about fruits and salad having a positive effect on PCOS.

The distribution of the study population according to the source of awareness of PCOS. Among those who were aware, majority of the population had awareness through textbook (58.33\%) and family $\&$ friends (16.66\%) about PCOS. Other sources of awareness included TV/Media, Internet and Medical personnel.

The distribution of the study population according to the knowledge about the causes of PCOS. There was an initial knowledge about dietary habits (74\%) 
being a cause of PCOS, post intervention there was an increase of $18 \%$ about the same. Knowledge about smoking and alcohol consumption was initially $65 \%$ which increased $10 \%$ post intervention. Majority of the study population had knowledge about sedentary lifestyle (60\%) and stressful lifestyle (75\%), post intervention there was an increase to $85 \%$ and $83 \%$ respectively.

The distribution of the study population according to the knowledge about the available treatment options for PCOS. The majority of the study population had knowledge about lifestyle changes (83\%), post intervention there was an increase of 7\%. Among the study population $35 \%$ had knowledge about Allopathic and $46 \%$ had knowledge about Ayurvedic treatment, post intervention to which there was an increase to $59 \%$ and $65 \%$ respectively.

The association between the knowledge about the etiology of PCOS with the awareness about the changes in body weight. Among the population, out of those who were aware about the etiology $45 \%$ felt there would be sudden increase in weight, $12.73 \%$ said there would be sudden decrease, $9 \%$ said there would be no change in weight whereas $32.72 \%$ felt there would be a gradual increase in the weight in PCOS. The association was found to be insignificant between the knowledge about etiology with the knowledge about changes in body weight in PCOS.

The association between the knowledge about the etiology of PCOS and the awareness about the mood swings in PCOS. Out of those who had knowledge about the etiology of PCOS $49.09 \%$ were aware about irritability and anger as being the mood change in PCOS, $12.72 \%$ felt depression, $14.5 \%$ felt anxiousness and $18.18 \%$ felt being easily fatigued as a type of mood change. The remaining felt there was no association of mood change with PCOS. The association was found to be insignificant between the knowledge about the etiology of PCOS and the knowledge about the mood changes in PCOS.

An association between the awareness about the changes in PCOS with the gender. Among the males, 92.15\% were aware about the changes in PCOS, and $7.85 \%$ were unaware. Among the females, 57.14\% were aware about the changes in PCOS whereas $42.86 \%$ were not. This is a significant association between awareness about changes in PCOS with gender.

The association between the knowledge about the lifestyle modifications in PCOS with the knowledge about the etiology of PCOS. Out of those aware about the etiology, $72.72 \%$ were aware about the effects of lifestyle modification in PCOS, whereas $27.28 \%$ were unaware. There is significant association between knowledge about lifestyle modifications in PCOS with the knowledge about etiology of PCOS. The knowledge about the complications of PCOS with the knowledge about the etiology of PCOS. Out of those aware about etiology of PCOS, 61.81\% had knowledge about the complications of PCOS, whereas, $38.19 \%$ were unaware about the knowledge of complications. There is significant association between the knowledge about complication of PCOS with the knowledge about etiology of PCOS.

The association between the knowledge about the positive diet in PCOS with the knowledge about the etiology of PCOS. From the 55\% of the study population that were aware about the etiology of PCOS, 18.18\% had knowledge about the positive effects of diet in PCOS, but in $81.82 \%$ the knowledge was absent. There was no significant association between the knowledge about the positive effect of diet in PCOS with the knowledge about etiology of PCOS.

The association between the knowledge about the complications in PCOS with the knowledge about the lifestyle modifications in PCOS. Among the population 
who has knowledge about the lifestyle modifications in PCOS, $57.83 \%$ were aware of the complications in PCOS whereas $42.16 \%$ were unaware. There was no significant association between the knowledge about complications of PCOS with the knowledge about the lifestyle modifications in PCOS.

\section{Discussion}

In the present study, we surveyed the knowledge among 100 students (51 Males and 49 Females) of Subharti Medical College about Polycystic Ovarian Syndrome, particularly on common symptoms, complications, source of knowledge, management and treatment options, influence of exercise and food upon PCOS, feelings and perception about the condition.

In our study, subjects were aged between 18-23 years old, $21 \%$ were in 18-19 age group, $73 \%$ were in 20-21 age group, $6 \%$ were aged in between 22-23 age group. Participants were undergraduate students of MBBS. An another study done by Sonia Rawat ${ }^{[7]}$ in 2017 consisted of participants aged 15-17 years $(48.9 \%)$ and $17-18$ years $(51.1 \%)$.

In our study, only $16 \%$ have minimal knowledge about the condition. $84 \%$ of the study population were highly aware of the reasons and consequences of the condition. A study done by Ansari et al ${ }^{[8]}$ in 2014 suggested that on symptoms and awareness of PCOS in urban Pakistani women found that only $10 \%$ were familiar with PCOS, which is much lower than our study. A study done by Sabitha \& Sunanda ${ }^{[9]}$ in 2016 performed in Nitte Usha Institute of Nursing Sciences, found $76 \%$ had average knowledge and $10.7 \%$ had good knowledge about PCOS. The percentage of acquaintance is a little bit lower than our value. A study done by Hansa et al ${ }^{[10]}$ in 2016 performed in a Semi Urban School of Sambalpur, India, identified that $78 \%$ students never heard of PCOS before, which is much higher than our value.
An another study done by Abbas et al., ${ }^{[11]} 2014$ in a study in Tertiary Care Hospitals of Pakistan, found $55.6 \%$ patients to be unaware about PCOS which is far different than our value.

In our study we found that $48 \%$ of participants had a perception about irregular periods being a symptom of PCOS, whereas Sreeraj et al. ${ }^{[12]}$ in 2016 showed that only $30 \%$ participants thought that irregular period was an important symptom of PCOS. This result was inconsistent with our study. In our study, participants also chose changes in scalp hair (51\%) and changes in body hair (53\%) as important signs and symptoms of PCOS. An another study done by Amal Alessa et al. ${ }^{[13]} 2017$ stated that $31.7 \%$ students chose hirsutism, and $18.9 \%$ chose frontal hair loss as a symptom.

In our study, among PCOS patients $10 \%$ of the population had information from medical personnel, $10 \%$ educated themselves through internet. A study done by Jayshree et al, ${ }^{[14]} 2017$ performed on polycystic ovarian syndrome in adolescents in Rotterdam showing that $11.5 \%$ of the population got knowledge from a doctor and 5\% learnt from the internet, which is consistent with our value.

Study of Sreeraj et al.(2016) in another study in Mumbai, India showed respondent's main source of information about PCOS as doctor and internet which account for $51 \%$ and $22 \%$ respectively. This study data is inconsistent with ours.

Anil et al. (2016) in a previous study of Mumbai also showed $81 \%$ participants think PCOS is manageable. In our study $82 \%$ expressed that it is a manageable condition. These results are similar to our study.

In our study, participants were asked if they thought different forms of exercises will help in coping with PCOS. 52\% agreed with exercise helping as a form of coping, out of which, 16\% said Yoga, 9\% 
said Gym, 12\% said alking/Running in the open, 10\% said playing a sport and 5\% chose dieting, whereas Sreeraj et al. (2016) stated in their study in India that $62 \%$ were aware of exercise helping in PCOS, however only half of them did exercise regularly. This result was higher compared to our study. Another study by Safa Jahangir, (2017) showed $74.86 \%$ participants agreed that exercise helps in managing PCOS. Regarding the effect of diet on PCOS, $40 \%$ of the participants chose fruits and salads, $16 \%$ chose egg and poultry and 10\% chose daal as having a positive influence on health in PCOS, whereas in a study conducted by Amal Alessa et al. (2017) 63.2\% chose fruits and vegetables and $74.6 \%$ chose protein rich foods to be having a positive effect on health in PCOS. These results were contrasting with our study.

About the knowledge of treatment methods of polycystic ovarian syndrome,

our study exhibited that most of the participants (83\%) thought lifestyle modification is the first choice of treatment after the audiovisual intervention. On giving an audiovisual presentation for 30 minutes about PCOS, this knowledge increased to $90 \%$. On the contrary, Anil et al. (2016) in a study found that lifestyle modification as a treatment option was selected by $24 \%$ population which is inconsistent to our study. Among the other treatment options exercise was chosen by $52 \%$ of population in our study. On the contrary, the study in Mumbai reveals $20 \%$ people thought exercise is a good treatment option for managing PCOS which is close to our data. Other participants chose Ayurvedic (46\%), Allopathic (35\%), Homeopathic (39\%), and Surgical measures (29\%). $17 \%$ of our participants thought that PCOS was non-manageable and Anil et al. 2016 study of Mumbai also showed 19\% participants think PCOS is non-manageable, which is similar to our study. According to our study results and the studies that are compared to it, we can conclude that more studies are needed to be conducted to establish a more clear comparison on various aspects regarding polycystic ovarian syndrome.

\section{Conclusion}

Among the study population, $84 \%$ of the students were aware about PCOS.

- Awareness about PCOS was seen more in boys (94\%) as compared to girls $(73.4 \%)$ out of the total number of boys and girls.

- In our study, awareness about PCOS was significantly higher in the students whose fathers were either graduate or above.

- In our study population almost $50 \%$ students had the knowledge about various changes in PCOS.

- $80 \%$ of the study population had the knowledge about the effect of physical exercise on PCOS.

- Among the students who had the knowledge about effect of exercise on PCOS, $77 \%$ of them preferred yoga.

- Textbooks were the source of awareness about PCOS in majority (49\%) of the students.

- Stressful lifestyle (75\%) and dietary habits (74\%) were considered as most important causes of PCOS by the students in our study population.

- $83 \%$ students among the study population had the knowledge about lifestyle changes as the most important among the available treatments for PCOS.

- Among the students who had the knowledge about etiology of PCOS, $62 \%$ also had the knowledge about complications of PCOS.

\section{Conflict of Interest: Nil}

Source of Funding: Nil

Ethical Clearance: Permission taken from 
departmental ethical Committee

\section{References}

1. Sedigheh Sedighi et al . Comparison of Lifestyle in Women With Polycystic Ovary Syndrome and Healthy Women. . Published online 2014 Aug 31.

2. A study on knowledge \& awareness of polycystic ovarian syndrome among female non medical undergraduate students. This dissertation is submitted to the Department of Pharmacy, East West University in the partial fulfillment of the requirements for the Degree of Bachelor of Pharmacy. ; Submitted To Tilka Fannana; Submitted By Safa Jahangir

3. Somaya Ouda Abd Elmenim\& Afaf Mohamed Mohamed Emam. Effect of Lifestyle Changes on Symptoms of Polycystic Ovarian Syndrome in Obese Girls. IOSR Journal of Nursing and Health Science (IOSR-JNHS)e-ISSN: 23201959.p- ISSN: 2320-1940 Volume 5, Issue 3 Ver. II (Mar. - Apr. 2016), PP 01-10

4. Polycystic ovarian syndrome (PCOS) linked to anxiety and depression. By MGH Centre for Women's Mental Health; January 26th, 2017

5. Jenni Glenn Gingery, Colleen Williams; Large-scale study links PCOS to mental health disorders; April 10, 2018.

6. PothirajPitchai, S. R. Sreeraj, ParmarReema Anil . Awareness of lifestyle modification in females diagnosed with polycysticovarian syndrome in India: explorative study

7. Sonia Rawat, Gomathi B., Laxmi Kumar, Mahalingam V., Structured teaching programme on knowledge about polycystic ovarian syndrome among adolescent girls, Himalayan college of nursing, SRHU, Dehradun, Uttrakhand, India.

8. Ansari, A., Gul, S., Zahid, S.A. (2014), PCOS: Symptoms and Awareness in Urban Pakistani Women, International Journal of Pharma Research and Health Sciences, [Online], [Accessed: 15th August 2017]

9. Sunanda B, Sabithya Nayak, study to assess the knowledge regarding PCOS among nursing students of NUINS, Paneer, Mangalore.

10. Hansa, J. Rajkumari, P., Sahoo, J., Sujata, P., Sahoo, G., (2016) Awareness about PCOS and the Likelihood of Its Symptoms in Adolescent Girls in a Semi-Urban Set-Up: A Cross Sectional Study. Journal of Medical science \& clinical research, 4(11), pp.12264

11. Abbas $\mathrm{N}$ et al. perception and attitude of patients regarding polycystic ovarian syndrome in tertiary care hospitals, Pakistan: A survey based study. International journal of pharmacy and therapeutics.

12. Pitchai P, Sreeraj, S.R. and Anil P.R, Awareness of lifestyle modification in females diagnosed with polycystic ovarian syndrome in India: Explorative study, international journal of reproduction, contraception, obstetric and gynaecology

13. Amal Alessa et al. Awareness of polycystic ovarian syndrome among Saudi females. International journal of medical science and public health.

14. Jayshree J.U., Chaitanya A. S. (2017). Awareness of PCOS (polycystic ovarian syndrome) in adolescent and young Girls. International Journal of Reproduction, Contraception, Obstetrics and Gynecology, 6(6). 